\title{
PrefIndex: An Efficient Supergraph Containment Search Technique
}

\author{
Gaoping Zhu, Xuemin Lin, Wenjie Zhang, Wei Wang, and Haichuan Shang \\ The University of New South Wales, Sydney, NSW, Australia \\ \{gzhu, l xue, zhangw, weiw, shangh\}@cse. unsw. edu.au
}

\begin{abstract}
Graphs are prevailingly used in many applications to model complex data structures. In this paper, we study the problem of supergraph containment search. To avoid the NP-complete subgraph isomorphism test, most existing works follow the filtering-verification framework and select graph-features to build effective indexes, which filter false results (graphs) before conducting the costly verification. However, searching features multiple times in the query graphs yields huge redundant computation, which leads to the emergence of the computation-sharing framework. This paper follows the roadmap of computation-sharing framework to efficiently process supergraph containment queries. Firstly, database graphs are clustered into disjoint groups for sharing the computation cost within each group. While it is shown NP-hard to maximize the computation-sharing benefits of a clustering, efficient algorithm is developed to approximate the optimal solution with an approximation factor of $\frac{1}{2}$. A novel prefix-sharing indexing technique, PrefIndex, is then proposed based on which efficient query processing algorithm integrating both filtering and verification is developed. Finally, PrefIndex is enhanced with multi-level sharing and suffix-sharing to further avoid redundant computation. An extensive empirical study demonstrates the efficiency and scalability of our techniques which achieve orders of magnitudes of speed-up against the state-of-the-art techniques.
\end{abstract}

\section{Introduction}

Recently, graph structured data have been increasingly adopted in applications such as Bio-informatics, Chemistry, Social Networks, WWW, etc. For instance, graphs are used to model protein interaction networks and chemical compounds in Bio-informatics and Chemistry, respectively. Efficient query processing is thus strongly demanded by graph database.

Graph containment search is defined as supergraph containment search [2] and subgraph containment search [12]. Given a query graph $q$ and a graph database $D=\left\{g_{1}, \ldots, g_{n}\right\}$, supergraph containment search finds all the graphs in $D$ contained by $q$, while subgraph containment search finds all the graphs in $D$ containing $q$. In Chemistry, given a newly found molecule (query graph) and a large number of descriptors (data graphs indicating chemical properties), we can predict its chemical function based on the descriptors it contains. In pattern recognition, given a graph structured background (query graph) and various

M. Gertz and B. Ludäscher (Eds.): SSDBM 2010, LNCS 6187, pp. 360-378, 2010.

(C) Springer-Verlag Berlin Heidelberg 2010 

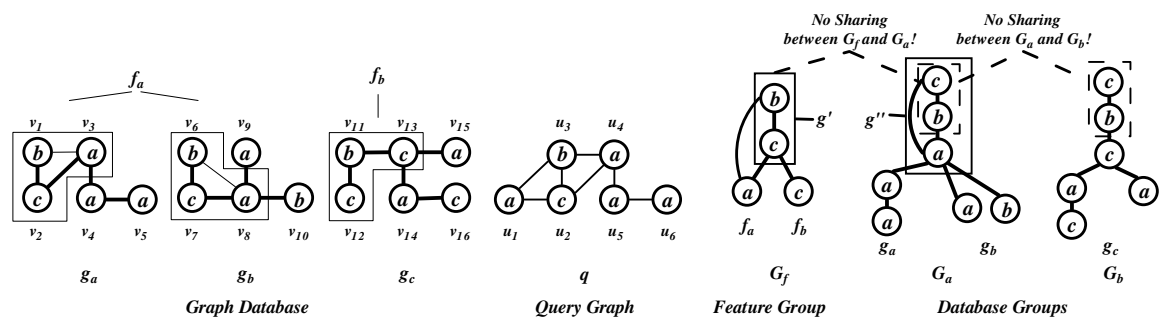

Fig. 1. Supergraph Containment Search

objects (data graphs), we may detect the foreground objects contained in the background. More applications can be found in [2, 12]. Regarding Figure 1, the result of supergraph containment search of query graph $q$ is $g_{a}$.

Since the subgraph isomorphism test is NP-complete [4], most works adopt the filtering and verification framework. While a feature-based index filters most false results in the filtering phase, survived candidate graphs are checked in the verification phase. Unlike its extensively studied dual version $[3,5,7,19,10,12$, 13, 16, 17], supergraph containment search is comprehensively investigated in only two studies 2, 14]. cIndex, proposed in [2], adopts historical query-log to select features for maximizing pruning power. Regarding Figure 1 assume that $f_{a}$ and $f_{b}$ are two features. cIndex first tests if $q$ contains $f_{a}$ and $f_{b}$. As $f_{b}$ is not contained by $q, g_{c}$ is filtered; while $g_{a}$ and $g_{b}$ survived to be candidates as $f_{a}$ is contained by $q$. As $f_{a}$ is a subgraph of $g_{a}$ and $g_{b}$, it will be searched for two more times in $q$ for the verifications of $g_{a}$ and $g_{b}$. Generally speaking, if a feature $f$ is contained by $n$ candidates, the subgraph isomorphism test on $f$ against $q$ will be repeated $(n+1)$ times (including one test in the filtering phase).

To avoid redundant subgraph isomorphism test cost, [14] proposes GPTree, a computation-sharing framework. It encodes each graph or feature in a sequence called GVCode. The GVCodes of a group of graphs or features are organized in a tree called GPTree such that a common subgraph of these graphs or features is stored only once as a prefix of the tree. This finally yields a forest structured database or index. GPTreeTest, the proposed subgraph isomorphism algorithm, verifies a group of graphs or features altogether by sharing the computation (subgraph isomorphism test) cost of the common subgraph within the group. For instance, in Figure 1, one feature group $G_{f}$ (for $f_{a}, f_{b}$ ), two database groups $G_{a}$ (for $\left.g_{a}, g_{b}\right)$ and $G_{b}$ (for $g_{c}$ ) are built. The computation cost of the common subgraphs $g^{\prime}$ and $g^{\prime \prime}$ are shared within $G_{f}$ and $G_{a}$, respectively. However, with further observations, GPTree has the following defects: (1)Computation cost can not be shared between filtering and verification, as the common edge $b-c$ of $G_{f}$ and $G_{a}$ can not be shared between them; (2)Computation cost can not be shared between database groups, as the common edge $b-c$ of $G_{a}$ and $G_{b}$ can not be shared between them; (3)GPTree prefers to select large-sized common subgraphs for sharing, which goes against the fact that large-sized subgraphs are usually infrequent and not likely to be shared by many graphs. 
Motivated by the above observations, this paper proposes a novel computationsharing framework with the aim to maximize the computation-sharing benefits. The main contributions of this paper are summarized as follows.

- We propose to cluster database graphs into disjoint groups such that graphs in each group contain a common feature $f$. While it is shown NP-hard to optimize the computation-sharing benefits, an efficient greedy algorithm is used to approximate the optimal solution with an approximation factor of $\frac{1}{2}$.

- Based on QuickSI traversal technique [9], a novel prefix-sharing indexing technique PrefIndex and a query processing algorithm PrefSearch are developed to share computation cost between filtering and verification.

- A group encoding technique is proposed to efficiently construct PrefIndex for a group of graphs based on the effective ordering of their GVCodes.

- Multi-level sharing and suffix-sharing techniques are proposed to enhance PrefIndex for sharing computation cost among database groups and further sharing computation within each database group, respectively.

Organization. The rest of the paper is organized as follows. Section 2 gives preliminaries and formalizes the problem. Section 3 presents the database clustering, feature selection and index construction techniques. Section 4 proposes our sharing-aware query processing algorithm integrating filtering and verification. Section 5 presents our multi-level sharing and suffix-sharing techniques. Experimental results and related work are reported in Section 6] and 7, while Section 8 concludes our study.

\section{Preliminaries}

\subsection{Problem Statement}

For presentation simplicity, our study only focuses on simple, vertex-labeled graphs. A simple graph is an undirected graph with no self-loops nor multiple edges between any two different vertices. From now on, a database graph is called a data graph, while a query graph is called a query. All data graphs are assumed to be connected. Nevertheless, our approach can be immediately extended to directed or edge-labeled graphs.

Given two sets of labels, $\Sigma_{V}$ and $\Sigma_{E}$, a graph $g$ is defined as a triplet $(V(g)$, $E(g), l)$ where $V(g)$ and $E(g)$ denote the vertex set and edge set of $g$, respectively. $l$ is a mapping: $V(g) \rightarrow \Sigma_{V}$ which assigns a label $l(u)$ to each vertex $u \in V(g)$.

Definition 1 (Subgraph Isomorphic). Given two graphs $g=(V, E, l)$ and $g^{\prime}=\left(V^{\prime}, E^{\prime}, l^{\prime}\right), g$ is subgraph isomorphic to $g^{\prime}$, denoted by $g \subseteq g^{\prime}$, if there is an injective function $f: V \rightarrow V^{\prime}$ such that (1) $\forall v \in V, f(v) \in V^{\prime}$ and $l(v)=l^{\prime}(f(v)) ;\left(\right.$ (2) $\forall(u, v) \in E,(f(u), f(v)) \in E^{\prime}$ and $l(u, v)=l^{\prime}(f(u), f(v))$. Under the above conditions, $g\left(g^{\prime}\right)$ is a subgraph (supergraph) of $g^{\prime}(g)$.

Definition 2 (Induced Subgraph). Given a graph $g$, a graph $g^{\prime}$ is an induced subgraph of $g$, if and only if (1) $g^{\prime}$ is subgraph isomorphic to $g$ under an injective function $f$; (2) $\forall u, v \in V\left(g^{\prime}\right)$, if $(f(u), f(v)) \in E(g),(u, v) \in E\left(g^{\prime}\right)$. 
Definition 3 (Supergraph Containment Search). Given a graph database $D=\left\{g_{1}, g_{2}, \ldots, g_{n}\right\}$ and a query graph $q$, find the answer set $D_{q}$ which consists of each $g_{i} \in D$ such that $g_{i} \subseteq q$.

\subsection{Computation-Sharing Framework}

cIndex [2] is the first filtering-verification framework for supergraph containment search. It applies the exclusive logic to filter data graphs; namely, if a feature $f \nsubseteq q$, any data graph $g$ such that $f \subseteq g$ can be filtered. However, sequentially testing each feature (candidate graph) against the query involves huge redundant computation cost in the filtering (verification) phase.

GPTree [14], the first computation-sharing framework, directly extends the filtering-verification framework to avoid the redundant computation cost in cIndex. Inspired by DFS code [1] and QISequence [9], GPTree proposes a new graph encoding technique called GVCode. Based on a spanning tree $t$ of a graph $g$, it encodes $g$ into a sequence represented by a regular expression Code C $_{g}=$ $\left[\left[S_{i} E_{i j}^{*}\right]^{V(g)}\right]$. Each entry $S_{i}$ is the mapped image of a vertex $v$ in $g$. While $S_{i} . l$ keeps the label of $v, S_{i} . p$ stores the entry corresponding to the parent vertex of $v$ in $t$. Once $S_{1}$ is fixed, $t$ is viewed as a tree rooted at the vertex corresponding to $S_{1}$ and thus $S_{1} \cdot p=0$. Each edge in $g$ but not in $t$ is recorded as a back edge. If $S_{i}$ has back edges, they are kept in $\left\{E_{i j}\right\}$.

Table1 1 gives two GVCodes of $g_{a}$ and $g_{b}$ in Figure1 The corresponding vertex of $S_{i}$ is in the bracket. The bold lines in Figure 1 show the spanning trees of $g_{a}$ and $g_{b}$. As $g^{\prime \prime}$ in Figure 1 is represented as a three-entry prefix from $S_{1}$ to $S_{3}$ in both Code $a$ and Code $e_{b}$, a tree structured organization of Code $e_{a}$ and $C_{\text {ode }}$, called GPTree, can be built to share the three-entry prefix as a common prefix. Note that a common prefix must be an induced subgraph of all graphs in the group and the GVCode of a graph is not unique.

Based on QuickSI [9], GPTreeTest, a new subgraph isomorphism test algorithm is proposed to verify a group of graphs sharing a common prefix. Regarding Table 1, GPTreeTest first searches in $q$ a subgraph isomorphism mapping of the common prefix from $S_{1}$ to $S_{3}$. A found mapping is then extended in a depth first fashion to search a whole mapping for the rest $C_{\text {ode }}$ and $C o d e_{b}$ under the common prefix, respectively.

Table 1. The GVCodes of $g_{a}$ and $g_{b}$

\begin{tabular}{|c|c||c|c|}
\hline Type & $\left(S_{i} . l, S_{i} \cdot p\right)$ & Type & $\left(S_{i} . l, S_{i} \cdot p\right)$ \\
\hline$S_{1}\left(v_{2}\right)$ & $(c, 0)$ & $S_{1}\left(v_{7}\right)$ & $(c, 0)$ \\
\hline$S_{2}\left(v_{1}\right)$ & $\left(b, S_{1}\right)$ & $S_{2}\left(v_{6}\right)$ & $\left(b, S_{1}\right)$ \\
\hline$S_{3}\left(v_{3}\right)$ & $\left(a, S_{2}\right)$ & $S_{3}\left(v_{8}\right)$ & $\left(a, S_{2}\right)$ \\
\hline$E_{31}$ & {$\left[e d g e: S_{3}\right]$} & $E_{31}$ & {$\left[e d g e: S_{3}\right]$} \\
\hline$S_{4}\left(v_{4}\right)$ & $\left(a, S_{3}\right)$ & $S_{4}\left(v_{10}\right)$ & $\left(b, S_{1}\right)$ \\
\hline$S_{5}\left(v_{5}\right)$ & $\left(a, S_{4}\right)$ & $S_{5}\left(v_{9}\right)$ & $\left(a, S_{1}\right)$ \\
\hline
\end{tabular}

Code $_{a}$

Code $_{b}$ 
The framework of GPTree can be outlined by four phases: (1) Mine frequent subgraphs from the database and build a feature-based index $\mathcal{F}=\left\{f_{1}, \ldots, f_{n}\right\}$. Each $f_{i}$ is attached a graph-ID list list $_{f_{i}}=\left\{g . i d \mid f_{i} \subseteq g \wedge g \subseteq D\right\}$. (2) Mine common induced subgraphs from features and data graphs respectively. Greedily select the largest common induced subgraphs and divide features and data graphs into disjoint groups such that each group $G$ shares a common induced subgraph $g^{\prime}$. Graphs in each group $G$ are encoded to share $C o d e_{g^{\prime}}$ as a common prefix, based on which a GPTree is built for $G$. (3) For filtering, each feature group is tested by GPTreeTest to obtain the candidate set $C_{q}=D-\bigcup_{f} \operatorname{list}_{f}(f \nsubseteq$ $q \wedge f \in \mathcal{F}$ ). (4) For verification, each database group is projected on $C_{q}$ and all non-empty projected database groups are verified by GPTreeTest to obtain the answer set $D_{q}$.

\section{PrefIndex}

Our Framework. We propose a novel computation-sharing framework called PrefIndex, which directly selects a feature as the common subgraph shared by a group of data graphs. Since a feature is encoded as a common prefix of all the data graphs in the group, its test cost can be shared between filtering and verification. The whole framework is outlined as follows.

1. Mine frequent induced subgraphs from the database and cluster all data graphs into disjoint groups $\left\{\left(f_{i}, G_{i}\right) \mid 1 \leq i \leq k\right\}$ such that for each graph $g$ in a group $G_{i}, f_{i}$ is an induced subgraph of $g$ and a selected feature.

2. Encode each graph $g$ in a group $G_{i}$ into Code $g$ of which $\operatorname{Code}_{f_{i}}$ is a prefix. Organize all GVCodes of graphs $G_{i}$ into a PrefIndex tree.

3. Apply our query processing algorithm integrating filtering and verification to process each group $G_{i}$ by sharing the computation cost of $\operatorname{Code}_{f_{i}}$.

\subsection{Cost Model and Feature Selection}

Given a data graph $g$ and a query $q$, if $f \subseteq g$, the subgraph isomorphism test cost of $g$ may be approximately represented by $\operatorname{cost}_{f}+\operatorname{cost}_{(g-f)}$. Given a group $G_{i}$ of graphs sharing a common induced subgraph $f_{i}$, if we test all graphs in $G_{i}$ by sharing the test cost of $f_{i}$ (to process $f_{i}$ only once), the cost gain (computationsharing benefits) approximately equals (11). Assume that no pre-knowledge is given on $q$, (1) may be interpreted as the expected gain for any $q$.

Given a database $D$ and a set $\mathcal{D}=\left\{\left(f_{i}, D_{i}\right) \mid 1 \leq i \leq m\right\}$ where each $D_{i}$ contains all graphs in $D$ which share $f_{i}$ as a common induced subgraphs, we call $f_{i}$ and $D_{i}$ master feature and master group of $f_{i}$, respectively.

Definition 4 (Disjoint Database Cover). Given a set $\mathcal{D}=\left\{\left(f_{i}, D_{i}\right) \mid 1 \leq\right.$ $i \leq m\}$ of master features and master groups and assume that $\bigcup_{i} D_{i}=D$, $\mathcal{G}=\left\{\left(f_{j}^{\prime}, G_{j}\right) \mid 1 \leq j \leq k\right\}$ is a disjoint cover of $D$ conforming $\mathcal{D}$ if and only if (1) $\forall\left(f_{i}^{\prime}, G_{i}\right), \exists\left(f_{j}, D_{j}\right) \in \mathcal{D}$ such that $f_{i}^{\prime}=f_{j}$ and $G_{i} \subseteq D_{j}$; (2) $\forall G_{j} \neq$ $G_{j}^{\prime}, G_{j} \cap G_{j}^{\prime}=\emptyset ;(3) \forall g \in D, \exists G_{j} \in \mathcal{G}$ such that $g \in G_{j}$. 
Given a disjoint cover $\mathcal{G}$ of $D$, the total cost gain by sharing the test cost of the master feature within each master group is in (2).

$$
\begin{gathered}
\operatorname{gain}_{G_{i}}=\operatorname{cost}_{G_{i}}-\operatorname{cost}_{G_{i}}^{\prime}=\left(\left|G_{i}\right|-1\right) \times \operatorname{cost}_{f_{i}} \\
\operatorname{gain}_{\mathcal{G}}=\sum_{i=1}^{k}\left(\left|G_{i}\right|-1\right) \operatorname{cost}_{f_{i}}
\end{gathered}
$$

Definition 5 (Maximized Gain (MG)). Given a database $D$ and a set $\mathcal{D}=$ $\left\{\left(f_{i}, D_{i}\right) \mid 1 \leq i \leq m\right\}$ of master features and master groups such that $\forall g \in D$, $\exists D_{i} \in \mathcal{D}, g \in D_{i}$, find a disjoint cover $\mathcal{G}$ of $D$ such that gain $\mathcal{G}$ is maximized.

Theorem 1. The problem of Maximized Gain is NP-hard.

Proof. In a special case of MG where each $\operatorname{cost}_{f_{i}}$ equals a constant $c$, gain $_{\mathcal{G}}=$ $c \times(n-k)$. Consequently, solutions of MG in this case aim to minimize $k$, which makes MG exactly a minimum set cover problem (NP-hard) [4].

Assume that each data graph contains at least one feature. Algorithm 1 is adopted to approximate the optimal disjoint cover with the maximum gain $_{\mathcal{G}}$. Let $\mathcal{G}$ be a set of already selected disjoint groups ( $\mathcal{G}$ is empty at the beginning). Let $g(\mathcal{G})$ be all the data graphs currently covered by $\mathcal{G}$. For each $D_{i}$ with a master feature $f_{i}$, let $\frac{\operatorname{cost}_{f_{i}} \times\left(\left|D_{i}-g(\mathcal{G})\right|-1\right)}{\left|D_{i}-g(\mathcal{G})\right|}$ be the average gain from each remaining data graph in $D_{i}-g(\mathcal{G})$. The greedy algorithm iteratively selects a group $\left(f_{i}, D_{i}-g(\mathcal{G})\right)$ with the highest average gain, until $\mathcal{G}$ covers all the data graphs.

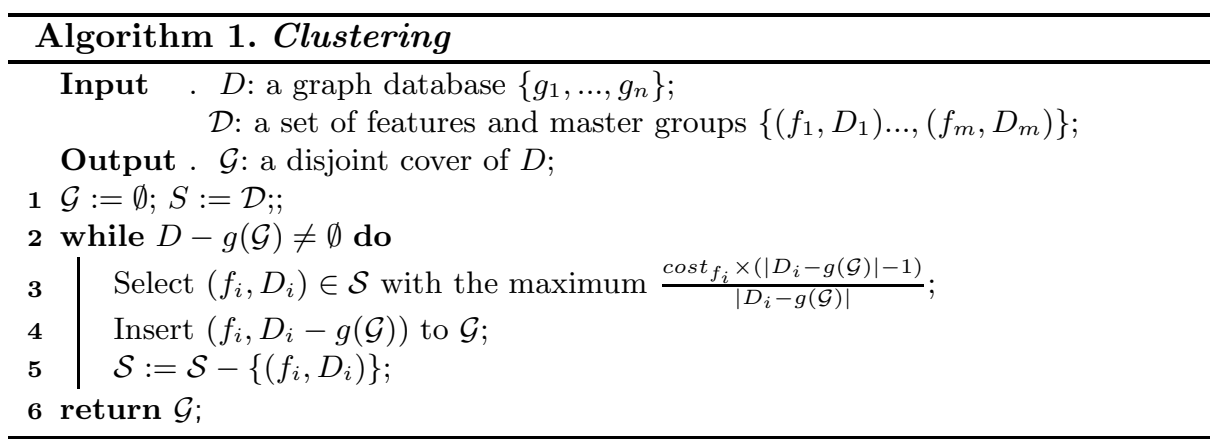

Example 1. Given $D=\left\{g_{1}, \ldots, g_{5}\right\}$ in Figure 2, for each $f_{i}$, its master group $D_{i}$ and cost $\operatorname{cost}_{f_{i}}$ are in the left two tables, respectively. Consider clustering (a) of PrefIndex by Algorithm 1]. For the first iteration, the average gains from $D_{1}$ to $D_{4}$ are $2,2, \frac{5}{2}$ and $\frac{8}{3}$, respectively. Consequently, $\left(f_{4},\left\{g_{1}, g_{4}, g_{5}\right\}\right)$ is selected. Then $g_{1}, g_{4}$ are removed from $D_{1}$, while $g_{5}$ is removed from $D_{3}$. The average gains of $D_{1}$ and $D_{3}$ become 0 , while the average gain of $D_{2}$ remains unchanged. For the second iteration, $\left(f_{2},\left\{g_{2}, g_{3}\right\}\right)$ is selected. Finally, we obtain a disjoint cover $\mathcal{G}=\left\{\left(f_{4},\left\{g_{1}, g_{4}, g_{5}\right\}\right),\left(f_{2},\left\{g_{2}, g_{3}\right\}\right)\right\}$ with a total gain of 12 .

Time Complexity. Due to the greedy nature of Algorithm 1, the worst case time complexity is $O\left(n^{2} \times m\right)$ where $n$ and $m$ are the numbers of data graphs and features, respectively. 


\begin{tabular}{|c|c|}
\hline$D_{1}$ & $g_{3}, g_{5}$ \\
\hline$D_{2}$ & $g_{2}, g_{3}$ \\
\hline$D_{3}$ & $g_{4}, g_{5}$ \\
\hline$D_{4}$ & $g_{1}, g_{4}, g_{5}$ \\
\hline
\end{tabular}

\begin{tabular}{|l|l|}
\hline $\operatorname{cost}_{\mathrm{f} 1}$ & 4 \\
\hline $\operatorname{cost}_{\mathrm{f} 2}$ & 4 \\
\hline $\operatorname{cost}_{\mathrm{f} 3}$ & 5 \\
\hline $\operatorname{cost}_{\mathrm{f} 4}$ & 4 \\
\hline
\end{tabular}

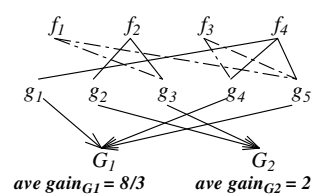

(a) PrefIndex Total Gain $=12$

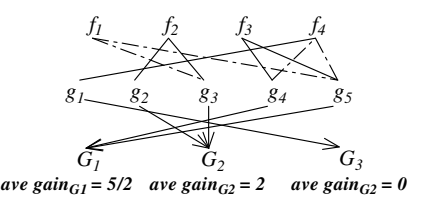

(b) GPTree Total Gain $=9$

Fig. 2. Example of Database Clustering

Accuracy Guarantee. Let $O P T$ and $A$ denote the total gains of the optimal solution and Algorithm 1, respectively. The following theorem can be proved.

Theorem 2. $A \geq \frac{1}{2} O P T$.

Proof. Let $\mathcal{G}=\left\{\left(f_{i}, G_{i}\right) \mid 1 \leq i \leq l\right\}$ be the optimal solution of MG and $O P T_{G_{i}}$ be the gain of group $G_{i}$; the total gain $O P T$ of $\mathcal{G}$ is (3). Let the number of graphs in $G_{i}$ be $n_{G_{i}}$; the average gain $a_{G_{i}}$ of $G_{i}$ is (4).

$$
\begin{gathered}
O P T=\sum_{i=1}^{l} \operatorname{cost}_{f_{i}} \times\left(\left|G_{i}\right|-1\right)=\sum_{i=1}^{l} O P T_{G_{i}} \\
a_{G_{i}}=\frac{\operatorname{cost}_{f_{i}} \times\left(n_{G_{i}}-1\right)}{n_{G_{i}}}
\end{gathered}
$$

Since the gain of any group with only one graph is 0 , we only consider those groups with at least two graphs. Let $\mathcal{G}^{\prime}=\left\{\left(f_{j}^{\prime}, G_{j}^{\prime}\right) \mid 1 \leq j \leq l^{\prime}\right\}$ be the greedy solution generated by Algorithm 1 and $A$ be the total gain of $\mathcal{G}^{\prime}$. Assume $G_{j}^{\prime}$ is selected at the $j$ th iteration. By removing any graph $g \notin G_{j}^{\prime}$ from $G_{i}$, we obtain $G_{i}^{j}=G_{i} \bigcap G_{j}^{\prime}\left(1 \leq j \leq l^{\prime}\right)$. By removing any group $G_{i}^{j}=\emptyset, G_{i}$ can be partitioned into a set of disjoint subgroups $\left\{G_{i}^{j_{1}}, \ldots, G_{i}^{j_{k}}\right\}$ such that $(1) \bigcup_{t=1}^{k} G_{i}^{j_{t}}=G_{i}$; (2) $\forall t, t^{\prime}\left(1 \leq t<t^{\prime} \leq k\right), j_{t}<j_{t^{\prime}}$.

Let the average gain of $G_{i}^{j_{t}}$ in the greedy algorithm be $a_{t}^{\prime}$ and the number of graphs in $G_{i}^{j_{t}}$ be $n_{t}^{\prime}\left(\sum_{t=1}^{k} n_{t}^{\prime}=n_{G_{i}}\right)$. Two observations can be made based on the partition of $G_{i}$. (1) Only the last subgroup $G_{i}^{j_{k}}$ may have a gain of 0 in the greedy solution, since $G_{j_{k}}^{\prime}$ may have only one graph and $G_{i}^{j_{k}}$ is not empty. If another group $G_{j_{t}}^{\prime}\left(j_{t}<j_{k}\right)$ has only one graph and $G_{i}^{j_{t}}$ is not empty, a new subgroup $G_{i}^{j_{k}} \cup G_{i}^{j_{t}}$ can be obtained to yield a gain greater than 0 . (2) Due to the greedy nature of Algorithm 1, $a_{1}^{\prime}$, the average gain of the first subgroup $G_{i}^{j_{1}}$ in the greedy solution, must be no less than $a_{G_{i}}$, Otherwise, $G_{i}$ instead of $G_{j_{1}}^{\prime}$ will be selected for the $j_{1}$ th iteration in the greedy solution. It can be concluded that the gain of $G_{i}^{j_{1}} \cup G_{i}^{j_{k}}$ in the greedy solution is at least $\frac{1}{2}$ of that in the optimal solution. For each rest subgroups $G_{i}^{j_{t}}(t \neq 1, k)$, due to the greedy nature of Algorithm 1, (5) can be immediately verified. By replacing cost $_{f_{i}}$ in (5) with (4), we have (6). Let the gain of $G_{i}$ in the greedy solution be $A_{G_{i}}$. By (6), we have (7), which leads us to our conclusion that $A \geq \frac{1}{2} O P T$.

$$
a_{t^{\prime}}^{\prime} \geq \frac{\operatorname{cost}_{f_{i} \times\left(n_{G_{i}}-\sum_{t=1}^{t^{\prime}-1} n_{t}-1\right)}}{n_{G_{i}}-\sum_{t=1}^{t^{\prime}-1} n_{t}}
$$




$$
\begin{gathered}
a_{t^{\prime}}^{\prime} \geq \frac{n_{G_{i}} \times\left(n_{G_{i}}-\sum_{t=1}^{t^{\prime}-1} n_{t^{\prime}}-1\right)}{\left(n_{G_{i}}-1\right) \times \sum_{t=1}^{t^{\prime}-1} n_{t}} \times a_{G_{i}} \geq \frac{1}{2} \times a_{G_{i}} \\
A_{G_{i}}=\sum_{t=1}^{k} a_{t}^{\prime} \times n_{t} \geq \sum_{t=1}^{k} \frac{a_{G_{i}} \times n_{t}}{2}=\frac{1}{2} O P T_{G_{i}}
\end{gathered}
$$

Remark. Generally speaking, the subgraph isomorphism test runs in an exponential time in the worst case and is algorithm, graph topology and graph size dependent. While our algorithm and its analysis apply to any given cost formula, $|V(f)|$ is used to approximate cost $_{f}$ in our implementation.

\subsection{Computation-Sharing Comparison}

On computation-sharing strategy, PrefIndex differs from GPTree in two ways. Firstly, GPTree selects common induced subgraphs for features and data graphs respectively, while PrefIndex directly selects common induced subgraphs of data graphs as features and shares the computation between filtering and verification. Secondly, GPTree greedily selects common induced subgraphs with the highest cost (largest size). Since larger subgraphs are usually unlikely to be contained by many graphs, PrefIndex uses a more natural heuristic to greedily select features with the highest average gain.

Consider clustering (b) of GPTree in Figure 2, though $f_{3}$ has the highest cost, it contributes less than $f_{4}$ as its master group has less data graphs, which only leads to a total gain of 8 . In our experiments, PrefIndex outperforms GPTree in all cases on computation-sharing benefits.

\subsection{Index Structure}

In PrefIndex, each feature $f$ is encoded into a common prefix of the GVCode of each graph $g$ in its master group $G_{f}$. This requires that $f$ must be an induced subgraph of each $g$ in $G_{f}$. We extend gSpan [11] to mine frequent, discriminative, induced subgraphs. $\mathcal{D}$ is initialized with the mined induced subgraphs and their master groups and then fed into Algorithm 1 for feature selection. Although a feature $f$ may be contained by a data graph $g$ not in $G_{f}$ as a non-induced subgraph, such information is not recorded in PrefIndex due to mining efficiency.

The index structure of PrefIndex is outlined as follows: (1) Given a disjoint cover $\mathcal{G}=\left\{\left(f_{i}, G_{i}\right) \mid 1 \leq i \leq k\right\}$ generated by Algorithm 1 encode each $g$ in a group $G_{i}$ into Code $_{g}$ by having $\operatorname{Code}_{f_{i}}$ as its prefix. In practice, QuickSI algorithm [9] is used to efficiently identify subgraph isomorphism mappings from $f_{i}$ to $g$. (2) Each entry $S_{i}$ of Code $g$ only stores the label of its corresponding vertex in $g$, its parent vertex in Code $_{g}$ and its back edge information. Figure 3(a) shows a PrefIndex of graphs $g_{a}$ and $g_{b}$ in Figure 1. The effective ordering of common prefixes and suffixes is discussed in the next section.

\section{PrefIndex Search}

This section firstly presents our querying algorithm for a group of graphs based on PrefIndex and then proposes two techniques to further enhance PrefIndex: (1) 


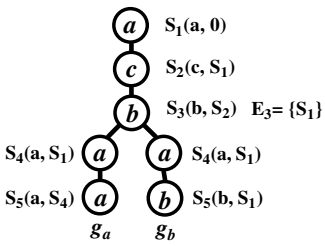

(a) PrefIndex $P I_{1}$ of $G_{I}$

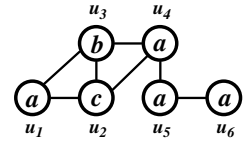

(b) Query Graph

\begin{tabular}{|c|c|c|c|}
\hline Type & $\left(S_{i} \cdot l, S_{i} \cdot p\right)$ & Type & $\left(S_{i} . l, S_{i} \cdot p\right)$ \\
\hline$S_{1}\left(v_{3}\right)$ & $(a, 0)$ & $S_{1}\left(v_{8}\right)$ & $(a, 0)$ \\
\hline$S_{2}\left(v_{2}\right)$ & $\left(c, S_{1}\right)$ & $S_{2}\left(v_{7}\right)$ & $\left(c, S_{1}\right)$ \\
\hline$S_{3}\left(v_{1}\right)$ & $\left(b, S_{2}\right)$ & $S_{3}\left(v_{6}\right)$ & $\left(b, S_{2}\right)$ \\
\hline$E_{31}$ & {$\left[e d g e: S_{1}\right]$} & $E_{31}$ & {$\left[\right.$ edge $\left.: S_{1}\right]$} \\
\hline$S_{4}\left(v_{4}\right)$ & $\left(a, S_{1}\right)$ & $S_{4}\left(v_{9}\right)$ & $\left(a, S_{1}\right)$ \\
\hline$S_{5}\left(v_{5}\right)$ & $\left(a, S_{4}\right)$ & $S_{5}\left(v_{10}\right)$ & $\left(b, S_{1}\right)$ \\
\hline \multicolumn{3}{|c}{$C o d e_{a}$} \\
\hline
\end{tabular}

Fig. 3. PrefIndex

Ordering GVCodes efficiently for PrefIndex; (2) Sharing pruning power among master groups. Given a disjoint cover $\mathcal{G}=\left\{\left(f_{i}, G_{i}\right) \mid 1 \leq i \leq k\right\}$, the PrefIndex of $G_{i}$ is denoted by $P I_{i}=\left\{\right.$ Code $\left._{g} \mid g \in G_{i}\right\}$. We aim to share the test cost of Code $_{f_{i}}$ between filtering and verification. $\operatorname{Code}_{f_{i}}$ is first processed to check whether a subgraph isomorphism mapping exists from $f_{i}$ to $q$. The test of $\operatorname{Code}_{f_{i}}$ is enforced to be conducted only once for $G_{i}$, which leads to the following fundamental theorem. The trivial proof is omitted here.

Theorem 3. If a query $q$ contains a data graph $g$, for each prefix Code' of

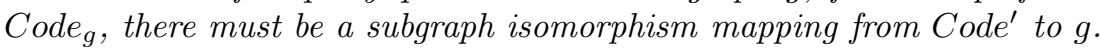

\subsection{Algorithm}

Algorithm Sketch. Based on Theorem 3, for each group $G_{i}$, our algorithm probes $P I_{i}$ in a depth first fashion. For each $C o d e_{g}$ in $P I_{i}$, once a mapping $\mathcal{P}^{\prime}$ is found from a prefix Code' of $\operatorname{Code}_{g}$ to $q$, the algorithm checks if $\mathcal{P}^{\prime}$ can be extended to cover the next vertex in Code $e_{g}$. If impossible, it backtracks in $P I_{i}$ to search the next mapping from $C_{0} e^{\prime}$ to $q$. Since $G_{i}$ may contain many graphs, the last vertex of $\operatorname{Code}_{f_{i}}$ may link to many suffix branches. The algorithm backtracks from the last vertex of $\operatorname{Code}_{f_{i}}$, if all suffix branches under this vertex have been explored in a depth first fashion. The algorithm terminates when no new mapping can be found for the first vertex of Code $_{f_{i}}$ or all GVCodes in $P I_{i}$ are detected to be subgraph isomorphic to $q$. The algorithm consists of two parts: PrefixQ and SuffixQ in Algorithm 2 and 3.

Example 2. Regarding the example in Figure 1, assume the database is clustered based on disjoint cover $\mathcal{G}=\left\{\left(f_{a}, G_{1}=\left\{g_{a}, g_{b}\right\}\right),\left(f_{b}, G_{2}=\left\{g_{c}\right\}\right)\right\}$. In Figure 3 , the two tables show the GVCodes of $g_{a}$ and $g_{b}$, while Figure 3 (a) and (b) show the PrefIndex of $G_{1}$ and the query.

In our algorithm, $S_{1}$ is first mapped to $u_{1}$ in $q$ as their labels match. $S_{2}$ is then mapped to $u_{2}$ as their labels and parents match; similarly, $S_{3}$ is mapped to $u_{3}$ as their labels, parents and back edges match $\left(S_{3} \cdot l=b, S_{3} \cdot p=S_{2}\right.$ and the back edge $\left(S_{3}, S_{1}\right)$ ). An intermediate mapping $\mathcal{P}_{1}$ from $\operatorname{Code}_{f_{a}}$ to $q$ is found. This corresponds to the filtering phase.

In verification phase, $\mathcal{P}_{1}$ is respectively extended for the suffixes of Code $_{a}$ and $\operatorname{Code}_{b}$. For both Code $_{a}$ and $\operatorname{Code}_{b}, \mathcal{P}_{1}$ fails to extend to $S_{4}$ as all adjacent vertices 
of $u_{3}$ are either already mapped or unable to meet $S_{4} \cdot p=S_{1}$. Consequently, $\mathcal{P}_{1}$ is abandoned and it backtracks from $S_{3}$ to search a new mapping. It then finds that no new mapping can be found for $S_{3}$ while keeping the existing mappings of $S_{1}$ and $S_{2}$. After it backtracks for one more depth, no new mapping can be found for $S_{2}$ while keeping the existing mapping of $S_{1}$. Finally, it starts from $S_{1}$ again and finds another intermediate mapping $\mathcal{P}_{2}$ from $\left\{S_{1}, S_{2}, S_{3}\right\}$ to $\left\{u_{4}\right.$, $\left.u_{2} u_{3}\right\}$. For Code $e_{a}, u_{5}$ and $u_{6}$ are mapped to $S_{4}$ and $S_{5}$ respectively, which verifies $g_{a}$ as an answer. For $\operatorname{Code}_{b}$, the extension fails at $S_{5}$. Since $\mathcal{P}_{2}$ is the last mapping from $C_{\text {ode }}$ to $q$, the query processing of $G_{1}$ terminates.

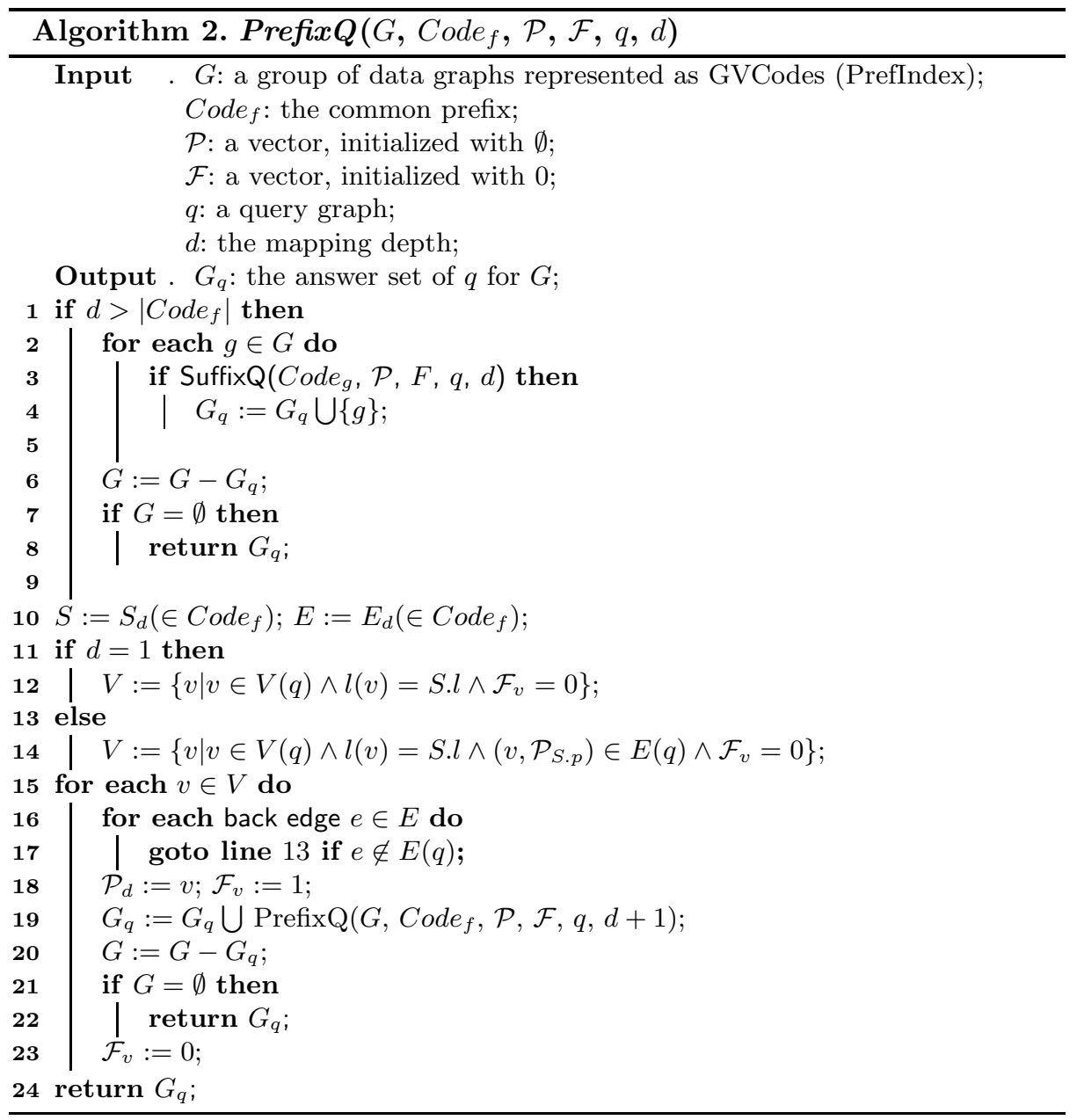

PrefixQ processes the common prefix Code $_{f}$ of a group $G$ and calls SuffixQ to complete the searching. It is in a recursive, depth first search fashion presented in Algorithm 2, The output $G_{q}$ is the answer set for $G$. $\mathcal{P}=\left\{\mathcal{P}_{1}, \mathcal{P}_{2}, \ldots\right\}$ stores 
the vertex mappings from $\operatorname{Code}_{f}$ to $q . \mathcal{P}_{d}=v_{i}$ means $S_{d} \in \operatorname{Code}_{f}$ is mapped to $v_{i}$ in $q . \mathcal{F}=\left\{\mathcal{F}_{1}, \ldots, \mathcal{F}_{|V(q)|}\right\}$ stores the vertex state for each $v_{i}$ in $q . \mathcal{F}_{i}=1$ means $v_{i}$ is already mapped to a vertex in $\operatorname{Code}_{f}$. The mapping depth $d$ indicates the current vertex $S_{d} \in$ Code $_{f}$ to be mapped. PrefixQ firstly checks if the current mapping $\mathcal{P}$ covers all the vertices of $\operatorname{Code}_{f}$. Condition $d>\mid$ Code $_{f} \mid$ (line 1) implies that a mapping $\mathcal{P}$ from Code $_{f}$ to $q$ is found. SuffixQ (to process each suffix respectively) is then called (line 3 ) to extend $\mathcal{P}$. Once a mapping from Code $_{g}$ to $q$ is successfully extended, $g$ is moved from $G$ to $G_{q}$. PrefixQ terminates when all GVCodes in $G$ are detected subgraph isomorphic to $q$ (line 7 ) or all mappings have been exhausted (line 13 and then line 22) for $S_{1}$ of Code $_{f}$.

SuffixQ processes a suffix under Code $_{f}$; namely, Code $e_{g}-C_{\text {Code }}$. It has the same input and also follows a recursive, depth first search fashion. The correctness of Algorithm 2 and 3 is immediate from Theorem 3 . Although costing exponential time in the worst case, they are very efficient in practice.

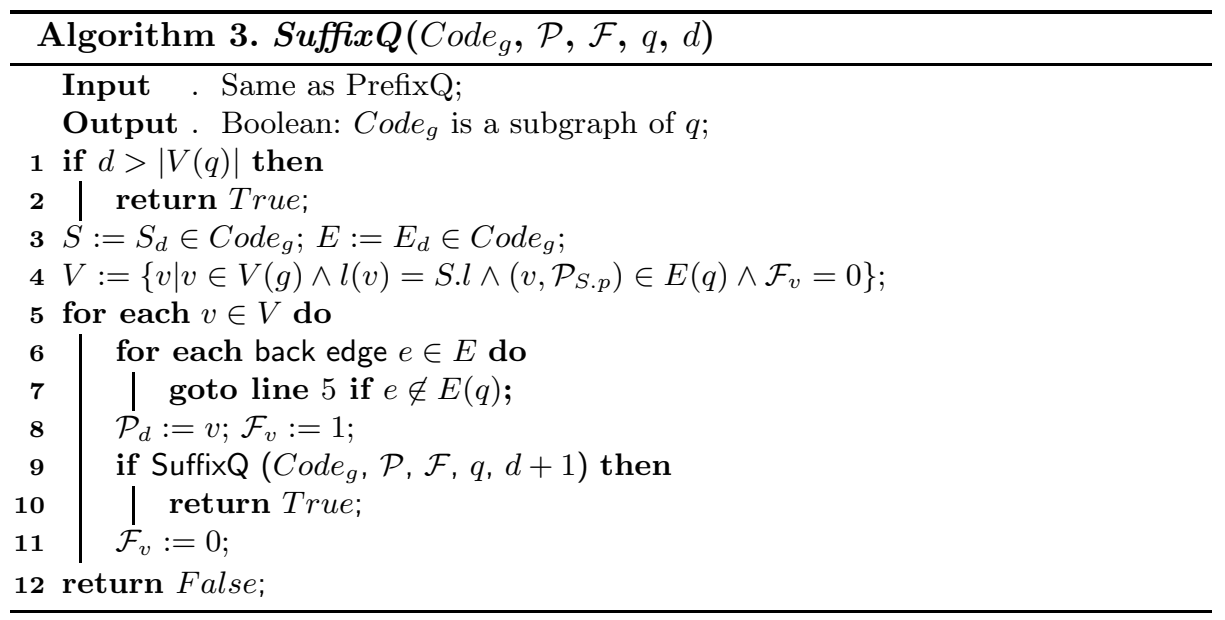

\subsection{Effectively Ordering GVCode}

Given a graph $g$ with $m$ vertices, there are $m$ ! different possible GVCodes. As shown in [9], a good ordering of a query $q$ can determine earlier if a subgraph isomorphism mapping from $q$ to a graph $g$ exists. Thus the edges (labels) in $q$ with lower occurrence rates should have a higher priority to be allocated earlier in $\mathrm{Code}_{q}$ to reduce the number of intermediate mappings to be considered. As our problem is the dual problem of that in [9], edges (labels) in a data graph $g$ with lower occurrence rates in the database are "signatures" of $g$ and should be allocated earlier in Code $_{g}$ for early pruning. When constructing PrefIndex $P I_{i}$ for a group $G_{i}$ with a feature $f_{i}$, QISequence ordering technique is firstly applied on $\operatorname{Code}_{f_{i}}$ and then on each suffix under $\operatorname{Code}_{f_{i}}$ in the same way. 


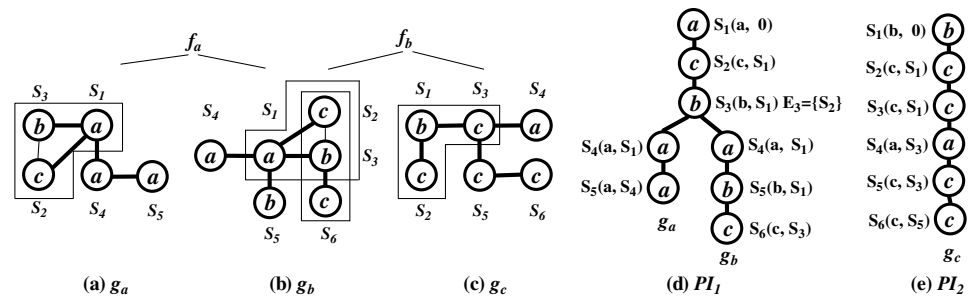

Fig. 4. Enhancing Pruning Power

\subsection{Enhancing Pruning Power}

In Figure 4, three graphs are clustered into two groups $G_{1}=\left\{g_{a}, g_{b}\right\}$ with $f_{a}$ and $G_{2}=\left\{g_{c}\right\}$ with $f_{b}$. Since $g_{b}$ contains $f_{b}, g_{b}$ can also be pruned if $f_{b}$ fails to pass the filtering phase. To share pruning power among different groups, two lists of graph IDs, M(Master)-List and R(Reference)-list are added at the last vertex of Code $_{i}$ in $P I_{i}$. M-list consists of IDs of the graphs in $G_{i}$, while R-List consists of IDs of the graphs not in $G_{i}$ but containing $f_{i}$. Regarding $G_{2}, M$-List $G_{2}=\left\{g_{c}\right\}$ and $R$-List $G_{2}=\left\{g_{b}\right\}$. When PrefixQ detects that $f_{b} \nsubseteq \nsubseteq q, g_{b}$ can be pruned from $G_{1}$ by not invoking SuffixQ on $g_{b}$. To realize this, the first SuffixQ call in each $G_{i}$ is enforced to happen only after PrefixQ calls on all groups are finished and a filtering list $R$ is obtained. In each survived $G_{i}$, SuffixQ is only invoked on those graphs not in $R$.

PrefixQ can be immediately modified to accommodate the above requirements. If PrefixQ reaches the depth $\mid$ Code $_{f} \mid+1$ of $G$ for the first time, SuffixQ is not invoked until the depth $\mid$ Code $_{f^{\prime}} \mid+1$ of all other $G^{\prime}$ is either reached for the first time (survived groups) or detected impossible to reach (R-lists of pruned groups are added to $R$ ). The modified algorithm is presented in Algorithm 4

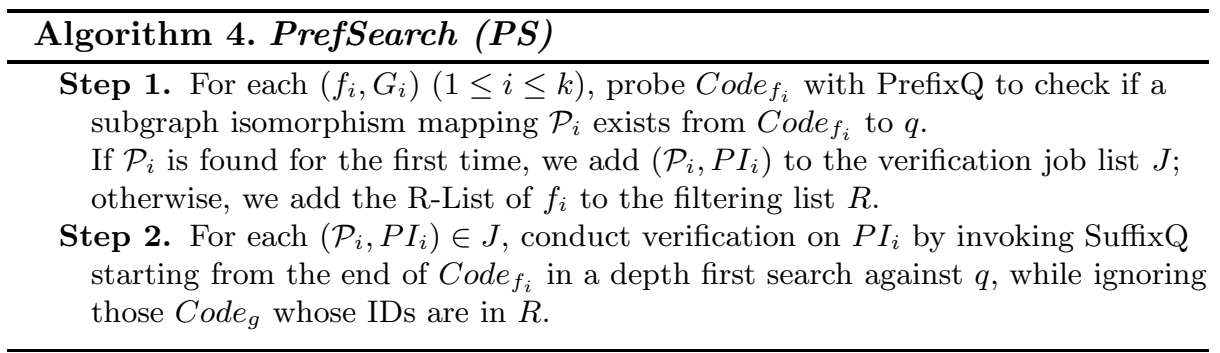

\section{Hierarchical PrefIndex Search}

This section explores two further computation-sharing opportunities missed by GPTree: (1) sharing computation among common prefixes of multiple groups; (2) sharing computation among multiple suffixes in each group. We propose to organize the PrefIndexes of multiple groups in a hierarchical structure. 


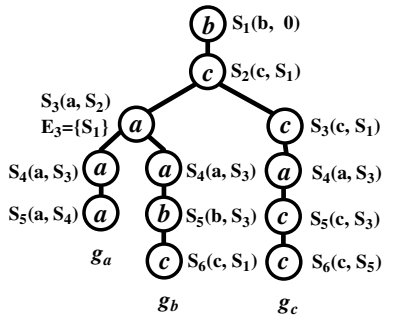

(a)

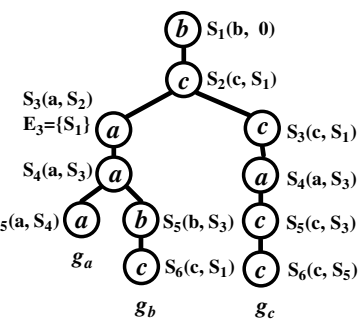

(b)

Fig. 5. Hierarchical PrefIndex

Multi-level Sharing. Regarding Figure 4, assume that (d) and (e) are the PrefIndexes of $G_{1}=\left\{g_{a}, g_{b}\right\}$ and $G_{2}=\left\{g_{c}\right\}$. Although $f_{a}$ and $f_{b}$ contain the same induced subgraph, an edge $(b, c)$, the chance to share it between $G_{1}$ and $G_{2}$ is missed since it is not the prefix of $P I_{1}$, To address this, a level 2 PrefIndex can be obtained by applying PrefIndex on all selected master features. Generally, a level $n$ PrefIndex can be obtained by applying PrefIndex on all sub-features of level $n-1$. The procedure can be iteratively performed until no new common subgraphs are identified at the current level.

Regarding Figures 4, we first mine frequent induced subgraphs from $f_{a}$ and $f_{b}$ as sub-features and then apply feature selection to construct a 2-level PrefIndex. Figure 5] (a) shows a 2-level PrefIndex where edge $(b, c)$ (sub-feature) is shared.

Suffix-Sharing. In PrefixQ, SuffixQ is recursively invoked for each survived suffix. Note that multiple suffixes in a group are still likely to share common entries. As in Figure 5 (a), two entries $\operatorname{Code}_{a} . S_{4}$ and $\operatorname{Code}_{b} . S_{4}$ have identical information as well as the common prefix. By sharing them as a common prefix of these two suffixes, the resulted PrefIndex is organized in Figure 5 (b).

Greedy algorithm and PrefIndex technique are adopted to explore the common prefixes of suffixes within each group. All possible next vertices in all suffixes which connects to the common prefix are identified and classified into different types based on label, parent and back edge information. We greedily selecting the vertex contained by the most suffixes and encode the vertex as the next entry of GVCodes of these suffixes. The greedy selection terminates when all types are contained by only one suffix. Frequent induced subgraph mining is not used here since the subgraph graph corresponding to each suffix is not always connected.

Building HiPrefIndex. PrefIndex technique is firstly applied on data graphs to build the first level index. Then we iteratively build index on common subfeatures identified on each next level. Finally, suffix-sharing is applied within each group to complete the hierarchical PrefIndex which is called HiPrefIndex. If the generated HiPrefIndex is forest structured, a dummy root, which links to the top of each PrefIndex, is inserted into HiPrefIndex.

Searching HiPrefIndex. The querying processing on HiPrefIndex starts from the (dummy) root of HiPrefIndex and probes HiPrefIndex in a depth first fashion. 
PrefixQ and SuffixQ can be immediately modified to support query processing on HiPrefIndex. For space limits, the details are not presented here.

Space-Time Efficiency vs Pruning Power. A branch in HiPrefIndex may correspond to more than one data graph. Regarding Figure 5 (b), the left branch of $\left(S_{3}, S_{4}\right)$ leads to $g_{a}$ and $g_{b}$. Given the query in Figure 1, the prefix corresponding to $f_{b}$ is not contained by $q$. Since we know $g_{b}$ also contains $f_{b}$, in order to remove $g_{b}$ in the filtering phase, we need to record graph IDs along each edge in HiPrefIndex to share pruning power. This increases not only the storage space but also the computation cost to check graph IDs on each edge. Thus we ignore such information and do not share pruning power in HiPrefIndex.

\section{Performance Evaluation}

We evaluate the performance of our techniques by comparing with GPTree. The following techniques are examined: (1) Indexing techniques PrefIndex and HiPrefIndex in Section 3 and 5. (2) Querying algorithms PrefSearch and HiPrefSearch in Section 4 and 5. (3) Querying algorithms GPTree(A) and GPTree(E) in 14]. GPTree(A) differs from GPTree(E) as it approximately mines frequent closed subgraphs to save index construction cost, which yields an (incomplete) feature set contained by the (complete) feature set of GPTree(E). We obtain the code of GPTree from its authors [14]. All algorithms are implemented in $\mathrm{C}++$ and compiled by GNU GCC. Experiments are conducted on PCs with Intel Xeon 2.4GHz dual CPU and 4G memory under Debian Linux.

Datasets: AIDS and AIDS10K. Two real datsets are used. AIDS Antiviral dataset, denoted by AIDS, contains 43,905 graph structured chemical compounds. It is a popular benchmark for studying graph queries downloaded from Development Therapeutics Program. To compare with GPTree based on its experiment settings, a subset of AIDS with 10K graphs, denoted by AIDS10K, is downloaded from http://www.xifengyan.net/software.htm.

Database and Query Set. For fair comparison, we adopt the experiment settings in [14]. We mine frequent subgraphs from AIDS10K with frequency ranging from $0.5 \%$ to $10 \%$ and randomly select $10 \mathrm{~K}$ graphs as the default database, while the default query set is exactly AIDS10K.

\subsection{Efficiency on Real Dataset}

The default database and query set is used to evaluate the efficiency of our techniques. The default query set is divided into 5 groups from $Q_{1}$ to $Q_{5}$. The answer set size of queries in $Q_{1}$ falls in $[0,20)$, while those of the rest groups from $Q_{2}$ to $Q_{5}$ fall in $[20,40),[40,60),[60,80)$ and $[80, \infty)$. Both techniques mine candidate features from the default database with a minimum frequency of $1 \%$. 
Table 2. Index Construction

\begin{tabular}{|c||c|c|c|c|}
\hline & PrefIndex & HiPrefIndex & GPTree(A) & GPTree(E) \\
\hline Index Construction (sec) & 311.5 & 313.6 & 129.7 & 697.9 \\
\hline Index Size (\# of Features) & 268 & 268 & 1301 & 1278 \\
\hline
\end{tabular}

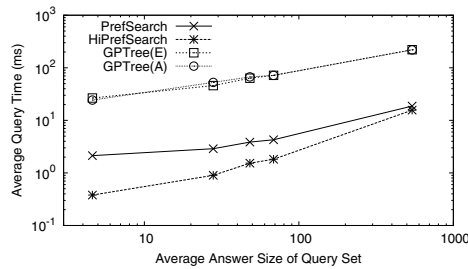

Fig. 6. Query Response

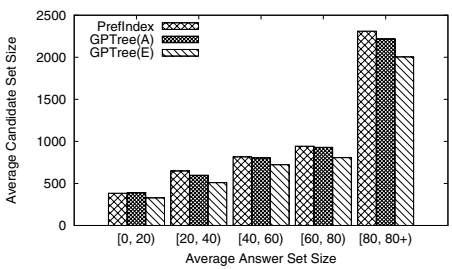

Fig. 7. Pruning Power

Query Processing. Figure 6] shows average query response time within each query groun 1 . PrefSearch and HiPrefSearch outperform GPTree(A) and GPTree(E) for up to 2 orders of magnitudes in query processing, while GPTree(E) slightly outperforms GPTree(A). Although HiPrefSearch disables some of its pruning power, it outperforms other techniques due to its multi-level and suffixsharing techniques.

Pruning Power. Figure 7 shows average pruning power measured by candidate size within each query group. The pruning power of PrefIndex is very similar to that of GPTree(E) and GPTree(A), while GPTree(E) outperforms GPTree(A) due to its complete frequent closed subgraph mining. Since HiPrefIndex disables a part of its pruning power to share more computation as discussed in Section 5. its pruning power is not evaluated here.

Index Construction. Table 2 shows index construction cost and index size measured by number of features. While most of the cost for both techniques is spent on the frequent subgraph mining, the effective ordering of GVCode of PrefIndex and HiPrefIndex only consumes less than $0.8 \%$ of the total cost. PrefIndex slightly outperforms HiPrefIndex due to the extra cost spent on mining multilevel subgraphs and common suffixes. GPTree(A) costs much less construction time as it approximately mine a small feature set. GPTree(E) costs the most index construction time since it mines not only a complete feature set but also common induced subgraphs from features and data graphs respectively. For fair comparison, we only focus on GPTree(E) for the rest experiments.

\subsection{Scalability on Real Dataset}

Varying Database Size. We first evaluate the scalability of our techniques by varying database size. For this reason, AIDS instead of AIDS10K is adopted to generate databases of various size. We first randomly select $10 \mathrm{~K}$ graphs from

${ }^{1}$ The $\mathrm{X}$-axis represents the average number of answer graphs in each group. 


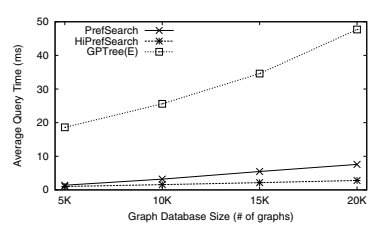

Fig. 8. Query Response

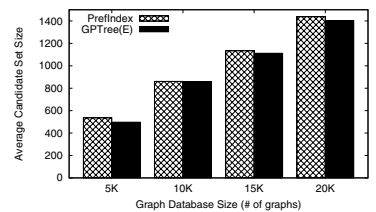

Fig. 9. Pruning Power

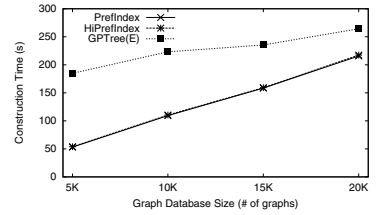

Fig. 10. Construction

AIDS as the query set from which we mine frequent subgraphs via the same way as in the overall performance and randomly select $5 \mathrm{~K}, 10 \mathrm{~K}, 15 \mathrm{~K}$ and $20 \mathrm{~K}$ frequent subgraphs to form 4 databases from $D_{1}$ to $D_{4}$.

Figure 8 shows average query response time for each database. The increment of query response time is almost linear for PrefSearch and HiPrefSearch with increasing database size. HiPrefSearch is still up to an order of magnitude faster than GPTree(E). Figure 9] shows average pruning power for each database. The pruning power of PrefIndex is similar to that of GPTree(E), which confirms the advantage of PrefSearch and HiPrefSearch over GPTree(E) mainly comes from the maximized computation-sharing benefits. Figure 10 shows index construction cost for each database. PrefIndex and HiPrefIndex always needs similar index construction time. It is because HiPrefIndex mines frequent subgraphs from a sequentially decreasing set of sub-features on each level, while common prefixes of suffixes are searched only within each group. GPTree(E) costs the most time due to the extra cost on mining common induced subgraphs.

Varying Data Graph Size. We then evaluate the scalability of our techniques by varying data graph size (in \# of vertices). We randomly select $10 \mathrm{~K}$ graphs from AIDS as the query set from which we mine frequent subgraphs via the same way as above and randomly select $5 \mathrm{~K}$ frequent subgraphs of 10 vertices as database $D_{1}$. We construct other four database from $D_{2}$ to $D_{5}$ by selecting $5 \mathrm{~K}$ frequent subgraphs of $12,14,16$, and 18 vertices respectively.

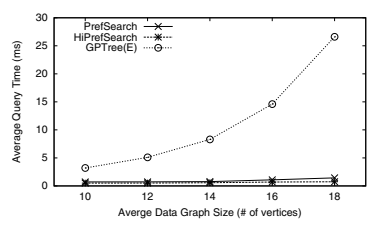

Fig. 11. Query Response

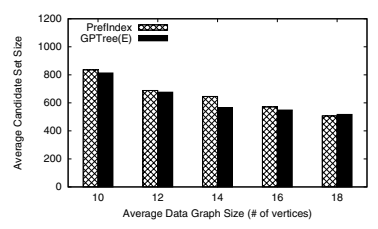

Fig. 12. Pruning Power

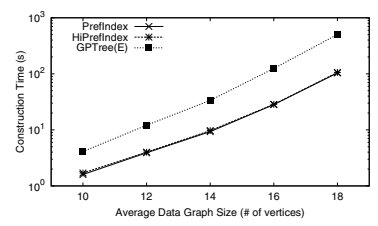

Fig. 13. Construction

The query response time, pruning power and index construction time for each database are respectively reported in Figures 11, 12 and 13. Note that the gap on query response time between PrefIndex and GPTree(E) are dramatically furthered with increasing data graph size, since the benefits of multi-level and suffix-sharing are more likely to be obtained on large graphs. While the pruning 
power for both techniques remains similar, index construction cost of PrefIndex and HiPrefIndex are very close and increase less significantly than GPTree(E).

\subsection{Scalability on Synthetic Dataset}

We evaluate the scalability on synthetic dataset by varying database size. A graph generator from [3] is used. A default query set of $10 \mathrm{~K}$ graphs is generated by setting the average graph size to 30 vertices, while the average density $\left(\frac{|V|}{|E|}\right)$ is set to 1.3. The distinct number of labels is set to 10 and distributed uniformly. The default database and 4 databases of $5 \mathrm{~K}, 10 \mathrm{~K}, 15 \mathrm{~K}$ and $20 \mathrm{~K}$ graphs are constructed in the same way as above.

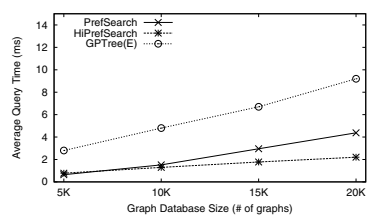

Fig. 14. Query Response

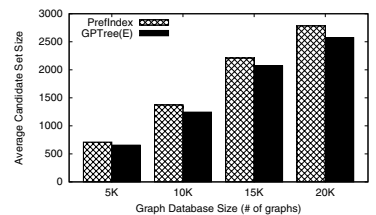

Fig. 15. Pruning Power

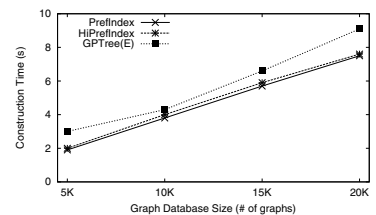

Fig. 16. Construction

The query response time, pruning power and index construction time are respectively recorded in Figure 14, 15] and [16. Although PrefSearch and HiPrefSearch outperform GPTree(E) on query response time and index construction cost, the gap between the techniques shortens a lot. Due the uniform distribution of vertex label, the number and size of frequent subgraphs greatly decrease and thus yields limited computation-sharing opportunity. However, PrefIndex still has its advantage over GPTree(E) as expected.

\section{Related Work}

Many studies have been done on graph containment search. While the subgraph containment search has been extensively studied, the supergraph containment search does not draw attentions from database community until most recently.

The filtering-verification framework is popular among most related work on subgraph containment search, which uses effective indexing techniques to filter most false results before the costly verification. Shasha et al. propose a pathbased index, GraphGrep [5], which is known as the first feature-based index for subgraph containment search. To enhance the pruning power, frequent subgraph mining techniques such as gSpan [11] and F3TM [15] are developed. Yan et al. develop an effective indexing approach gIndex [12] based on frequent, discriminative subgraphs. Due to the expensive cost of frequent subgraph mining, Zhang et al. and Zhao et al. propose TreePI [13] and $($ Tree $+\Delta)$ [16] independently to index frequent subtrees. Cheng et al. propose a verification-free framework FGIndex [3] to further avoid subgraph isomorphism test. Besides the feature-based 
index approaches, He et al. propose a clustering-based approach, called C-tree [6], to index graph closures (integration of graphs) in a B-tree like structure. It is the first work to support both exact and similarity subgraph containment search. Other clustering-based approaches include [8] and [1]. Moreover, Williams et al. [10] focus on the efficiency of processing small data graphs, while Jiang et al. [7] convert subgraph containment search to a string search problem. Recently, Shang et al. 9] present an efficient verification algorithm QuickSI.

On supergraph containment search, the first work cIndex proposed by Chen et al. 2] adopts historical query-log information to select features with maximized pruning power. Zhang et al. propose GPTree [14], a computation-sharing framework to share computation cost respectively in the filtering phase and verification phase. To address the defects of GPTree, our techniques propose efficient clustering and query processing algorithm to further share computation cost between filtering and verification, while multi-level and suffix-sharing techniques provide other opportunities to avoid redundant computation.

\section{Conclusions}

In this paper, a novel computation-sharing framework is proposed for supergraph containment search. All data graphs are clustered into disjoint groups for computation-sharing within each group. While the optimization problem MG is shown NP-hard, efficient greedy heuristic is used to approximate the optimal solution with an approximation factor of $\frac{1}{2}$. Based on the compact index structure, PrefIndex, an efficient algorithm PrefSearch integrating filtering and verification is proposed. PrefIndex is enhanced with multi-level sharing and suffix-sharing techniques to explore further sharing opportunities. An extensive empirical study demonstrates the efficiency and scalability of our proposed techniques which achieve orders of magnitudes of speed-up against the state-of-the-art techniques.

\section{References}

1. Berretti, S., Bimbo, A.D., Vicario, E.: Efficient matching and indexing of graph models in content-based retrieval. IEEE Trans. Pattern Anal. Mach. Intell. 23(10), 1089-1105 (2001)

2. Chen, C., Yan, X., Yu, P.S., Han, J., Zhang, D.-Q., Gu, X.: Towards graph containment search and indexing. In: VLDB, pp. 926-937 (2007)

3. Cheng, J., Ke, Y., Ng, W., Lu, A.: Fg-index: towards verification-free query processing on graph databases. In: SIGMOD Conference, pp. 857-872 (2007)

4. Garey, M.R., Johnson, D.S.: Computers and Intractability: A Guide to the Theory of NP-Completeness. W. H. Freeman, New York (1979)

5. Shasha, D., Wang, J.T.-L., Giugno, R.: Algorithmics and applications of tree and graph searching. In: PODS, pp. 39-52, 200

6. He, H., Singh, A.K.: Closure-tree: An index structure for graph queries. In: ICDE, p. 38 (2006)

7. Jiang, H., Wang, H., Yu, P.S., Zhou, S.: Gstring: A novel approach for efficient search in graph databases. In: ICDE, pp. 566-575 (2007) 
8. Messmer, B.T., Bunke, H.: A decision tree approach to graph and subgraph isomorphism detection. Pattern Recognition 32(12), 1979-1998 (1999)

9. Shang, H., Zhang, Y., Lin, X., Yu, J.X.: Taming verification hardness: an efficient algorithm for testing subgraph isomorphism. PVLDB 1(1), 364-375 (2008)

10. Williams, D.W., Huan, J., Wang, W.: Graph database indexing using structured graph decomposition. In: ICDE, pp. 976-985 (2007)

11. Yan, X., Han, J.: gspan: Graph-based substructure pattern mining. In: ICDM, pp. 721-724 (2002)

12. Yan, X., Yu, P.S., Han, J.: Graph indexing: A frequent structure-based approach. In: SIGMOD Conference, pp. 335-346 (2004)

13. Zhang, S., Hu, M., Yang, J.: Treepi: A novel graph indexing method. In: ICDE, pp. 966-975 (2007)

14. Zhang, S., Li, J., Gao, H., Zou, Z.: A novel approach for efficient supergraph query processing on graph databases. In: EDBT, pp. 204-215 (2009)

15. Zhao, P., Yu, J.X.: Fast frequent free tree mining in graph databases. In: ICDM Workshops, pp. 315-319 (2006)

16. Zhao, P., Yu, J.X., Yu, P.S.: Graph indexing: Tree + delta $\geq$ graph. In: VLDB, pp. 938-949 (2007)

17. Zou, L., Chen, L., Yu, J.X., Lu, Y.: A novel spectral coding in a large graph database. In: EDBT, pp. 181-192 (2008) 